Ibn Al-Haitham Jour. for Pure \& Appl. Sci. 34 (2) 2021

Ibn Al Haitham Journal for Pure and Applied Science Journal homepage: http://jih.uobaghdad.edu.iq/index.php/j/index

\title{
Analytical Study of Soret and Dufour effect in the Electro-osmotic peristaltic flow of Rabinowitsch fluid model
}

\author{
Saba S. Hasen \\ sabaoday79@gmail.com \\ Department of Mathematics, College \\ of Sciences, University of Baghdad, \\ Baghdad, Iraq
}

\author{
Ahmed M. Abdulhadi \\ ahm6161@yahoo.com \\ Department of Applied Science, \\ University of Technology, Baghdad,
}

Iraq

Article history: Received, 15,June,2020, Accepted29,November,2020, Published in April 2021

Doi: 10.30526/34.2.2629

\begin{abstract}
The present paper concerned with study the of combined electro-osmotic peristaltic transport with heat and mass transfer which is represented by the Soret and Dufour phenomenon with the presence of the Joule electrothermal heating through a microchannel occupy by Rabinowitsch fluid. The unsteady two-dimensional governing equations for flow with energy and concentration conservation have been formed in a Cartesian coordinate system and the lubrication theory is applied to modify the relevant equations to the problem. The Debye-Hukel linearization approximation is utilizing to modify the electrohydrodynamics problem. The expressions for the axial velocity, the temperature profile, the concentration profile, and the volumetric flow rate are obtained analytically to gain exact solutions, while the numerical integration is used to analyze the pressure rise. The attitude of the Helmholtz-Smoluchowski velocity, the electro-osmotic parameter, the Joule electrothermal heating, the Dufour number, the Soret number, is studied and graphically analyzed. It is showed that the presence of the electric field increased the velocity of the fluid and pressure rise, and it is revealed that the temperature and concentration profile congregate in the center of the channel with increase in the Soret and Dufour number.
\end{abstract}

Keyword: Peristaltic flow, Electro-osmosis, Axial electric field, Rabinowitsch fluid, Soret and Dufour effect, Joule heating.

\section{Introduction}

For many times, attention has been paid to studying a mechanism called peristalsis, which is a special type of fluid transport, it is created by the propagation of expansion or contraction progressive waves along the flexible wall of the conduit. Peristaltic mechanics find its 
importance in biological science in a general way and hydrodynamics in (an especial way). The literature detects that various studies have illustrated the phenomenon under diverse influences. Numerous researches are cited in [1-5].

Electro-osmosis is defined as the movement of an ionized liquid relative to a stationary charged superficies by means of an applied electric potential. The Electro-osmosis field allows the fluid infusion and control of flow using external electric fields, rescinding the requirement for mechanical pumps or valves with moving components. The velocities of flow correlating for electro-osmosis are not controlled by the size of the channel, with condition that the electrical double layer (EDL) is much smaller than the distinctive length scale of the channel. Electro-osmosis is considered a significant phenomenon in many biological and medical processes, where it has been invested in techniques of industrial separation in biotechnology and especially in medical micro-pumps, which have become common in microfluidics, the designs of electro-osmotic can produce considerable pressures and flow with no need to moving parts of mechanical and also in devices of capillary electrophoresis, pumping of the electro-osmotic can fulfill high effectiveness in capillaries lower than $100 \mathrm{~lm}$ and this is advantageous to deployment in systems of miniaturized chemical analysis. The pumps Electro-osmotic have analogous features to the pumps of electrohydrodynamic (EHD) and the pumps of a traveling wave, where the electrical force imposed generate the influence of pumping with no need for any mechanical parts and Thus, maintenance and replacement of other parts can be alleviated. The continuous amelioration in the design of the electro-osmotic pump has stimulated great attention in both experimental prototype testing and also computational and mathematical modeling. These two approaches have confirmed to be extremely important and complimentary in accelerating the appearance of next-generation electro-osmotic micro-pumps.

In the last few decades, literature has been made on electro-osmotic peristaltic transports. Pumps of the peristaltic when actuated associate with the electrostatic process achieves better results. Peristalsis with Electroosmotic pumping grants us an enormous range of applications in every area. Several mathematical models with different physical applications and diverse flow systems have been investigated. Chakraborty [6] is one of the first investigations in this field, as he has been proven that the axial electric field can raise the rates of microfluidic transport in the peristaltic pumps of in the microtubules. Tripathi et al. [7] discussed the effect of the external electric field on the Newtonian model with peristaltic flow through the microchannel and they concluded that the applied electric field can control the velocity and pressure gradient of the fluid during the flux, however, the impact of Joule electrothermal heating and buoyancy on nanofluids in a two-dimensional microchannel under electroosmotic peristaltic pumping is investigated in Refs.[8,9]. Further studies in this field are cited in [1013]. While Prakash et al. [14] studied the electroosmotic peristaltic transport impact on biorheological fluid in the microchannel with consideration of the existence of Joule heating, convective boundary condition, and thermal radiation. Investigations of the influence of electro-magnetohydrodynamics (EHD) characteristics together with heat transfer have been reported in Refs. [15-18]. Some other praiseworthy investigation regarding the topic can be seen via Refs. [19-25].

Heat transfer Occupies a substantial role in the cooling processes of medical and industrial applications and is currently deemed as a significant field of study since is a very important heat transfer in the human body. Also, heat transfer includes many intricate processes like 
evaluating skin burns to an organism, the destruction of unwanted tissues as a result of cancer, the technique of dilution in check blood flow, making of paper, processing of food, vasodilation, and radiation between the surface and its environment. Mass transfer is another substantial phenomenon in industry and physiology. It has numerous applications such as the process of membrane separation, diffusion of nutrients' out from the blood to neighboring tissues, osmosis of reverse, the process of distillation, the process of combustion, and chemical impurities diffusion. Several attempts can be viewed in the available reference list [26-34].

This paper displays a new hydrodynamic model to simulate the effect of the external electric field and the electric double layer thickness (EDL) on unsteady peristaltic transport of Rabinowitsch fluid through a symmetric microchannel with consideration the influence of Soret and Dufour phenomenon. Also, the effect of Joule electrothermal heating. Accordingly, the paper is organized as follows: in Section. 2, the mathematical model is formulated for this problem and The exact solution for the axial velocity, the temperature profile, concentration, and the volumetric flow rate is offered, whereas resort to a numerical integration to find the pressure rise. In Section.3, the impacts of the physical parameters are examined in detail via graphical representations. Finally, in Section 4, Key observations of the results are concluded.

\section{Modeling}

Let's start with the movement of the Non-Newtonian Rabinowitsch fluid model subject to the electroosmotic peristaltic transport through two-dimensional a symmetric horizontal microchannel of width $2 a$ as is illustrated in Figure (1). The fluid is under the influence of heat and mass transfer, with the interest in studying the effect of the Sort and Dufour phenomenon. The flow results from a sinusoidal peristaltic wave along the channel with wave speed $c$ and wavelength $\lambda$. The Cartesian coordinates were used to investigate the problem where the wave propagates parallel to the $X$-direction while $Y$-axis represented the perpendicular to the wave .

The mathematical formula of the channel can be expressed as follows

$\bar{Y}= \pm \bar{H}(\bar{X}, \bar{t})= \pm\left(a-\bar{\varphi} \cos ^{2} \frac{\pi}{\lambda}(\bar{X}-c \bar{t})\right)$

Where $a$ represents the half-width of the channel, $\bar{\varphi}$ is the wave amplitude, $c$ wave speed, $\bar{t}$ is the time and $\bar{H}(\bar{X}, \bar{t})$ the transverse displacement of the upper and lower wall respectively.

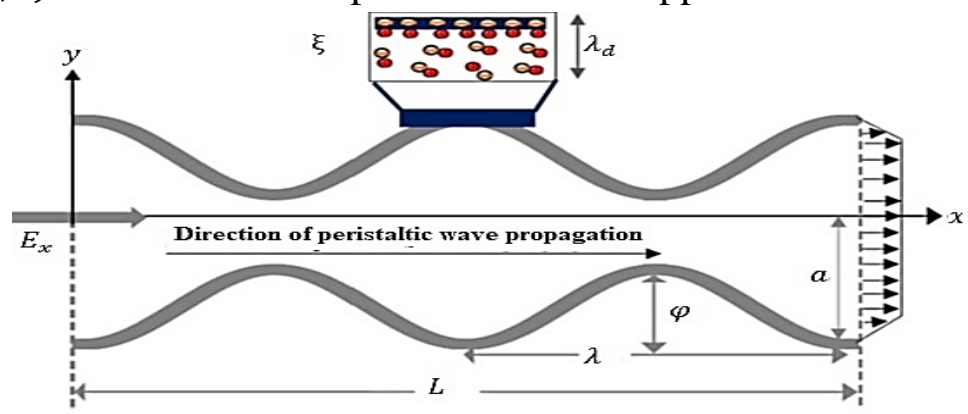

Figure 1. The geometry of the channel 
The governing equations for unsteady flow in two dimensional of incompressible nonNewtonian Rabinowitsch fluid under an axially applied electro kinetic body force in the laboratory frame $(\bar{X}, \bar{Y}, \bar{t})$ can be reviewed as follows.

$\frac{\partial \bar{U}}{\partial \bar{X}}+\frac{\partial \bar{V}}{\partial \bar{Y}}=0$

$\rho\left(\frac{\partial \bar{U}}{\partial \bar{t}}+\bar{U} \frac{\partial \bar{U}}{\partial \bar{X}}+\bar{V} \frac{\partial \bar{U}}{\partial \bar{Y}}\right)=-\frac{\partial \bar{P}}{\partial \bar{X}}+\frac{\partial \bar{S}_{\bar{X} \bar{X}}}{\partial \bar{X}}+\frac{\partial \bar{S}_{\bar{X} \bar{Y}}}{\partial \bar{Y}}+\bar{\rho}_{e} \bar{E}_{\bar{X}}+\rho g B_{T}\left(\bar{T}-\bar{T}_{0}\right)+\rho g B_{c}\left(\bar{C}-\bar{C}_{0}\right)$

$\rho\left(\frac{\partial \bar{V}}{\partial \bar{t}}+\bar{U} \frac{\partial \bar{V}}{\partial \bar{X}}+\bar{V} \frac{\partial \bar{V}}{\partial \bar{Y}}\right)=-\frac{\partial \bar{P}}{\partial \bar{Y}}+\frac{\partial \bar{S}_{\bar{Y} \bar{X}}}{\partial \bar{X}}+\frac{\partial \bar{S}_{\bar{Y} \bar{Y}}}{\partial \bar{Y}}$

$\rho c_{p}\left(\frac{\partial \bar{T}}{\partial \bar{t}}+\bar{U} \frac{\partial \bar{T}}{\partial \bar{X}}+\bar{V} \frac{\partial \bar{T}}{\partial \bar{Y}}\right)=\kappa\left(\frac{\partial^{2} \bar{T}}{\partial \bar{X}^{2}}+\frac{\partial^{2} \bar{T}}{\partial \bar{Y}^{2}}\right)+\frac{D K_{T}}{C_{S}}\left(\frac{\partial^{2} \bar{C}}{\partial \bar{X}^{2}}+\frac{\partial^{2} \bar{C}}{\partial \bar{Y}^{2}}\right)+\sigma \bar{E}_{\bar{X}}^{2}$

$\left(\frac{\partial \bar{C}}{\partial \bar{t}}+\bar{U} \frac{\partial \bar{C}}{\partial \bar{X}}+\bar{V} \frac{\partial \bar{C}}{\partial \bar{Y}}\right)=D\left(\frac{\partial^{2} \bar{C}}{\partial \bar{X}^{2}}+\frac{\partial^{2} \bar{C}}{\partial \bar{Y}^{2}}\right)+\frac{D K_{T}}{T_{m}}\left(\frac{\partial^{2} \bar{T}}{\partial \bar{X}^{2}}+\frac{\partial^{2} \bar{T}}{\partial \bar{Y}^{2}}\right)$

And the constituent equation for the Rabinowitsch fluid model is formulated as follows:

$\bar{S}_{\bar{X} \bar{Y}}+\bar{\zeta} \bar{S}_{\bar{X} \bar{Y}}=\mu \frac{\partial \bar{U}}{\partial \bar{Y}}$

where $\rho$ the density of the fluid, $\bar{U}, \bar{V}$ are the axial velocity and transverse velocity respectively, $\bar{P}$ is the pressure, $g$ is the gravitational acceleration, $B_{T}$ is the coefficient of thermal expansion, $\bar{T}$ is temperature, $B_{C}$ is the coefficient of concentration expansion, $\bar{C}$ is the concentration, $c_{p}$ is the specific heat at the constant pressure, $\kappa$ is thermal conductivity, $K_{T}$ is the thermal-diffusion ratio, $\sigma$ is electrical conductivity, $C_{S}$ is the concentration susceptibility, $T_{m}$ is the mean temperature. And $\bar{\rho}_{e}$ and $\bar{E}_{\bar{X}}$ are denote to the electric field and the net charge density in a unit volume of the fluid respectively. the Poisson's-Boltzmann equation for the electrical potential is used due to the electric double layer in the microchannel and is expressed as

$\nabla^{2} \bar{\Phi}=-\frac{\bar{\rho}_{e}}{\varepsilon_{0}}$

where the charge density is given by

$\bar{\rho}_{e}=e z\left(\bar{n}^{+}-\bar{n}^{-}\right)$

Where $e$ is the electronic charge, $z$ is charge balance, $\bar{n}^{+}$and $\bar{n}^{-}$are the number of densities of positive (cations) and negative (anions) respectively. To obtain the potential distribution it necessary to determine the charge number density, therefore Nernst-Planck equation [35] useful for this purpose, which can represent as follows

$\left(\frac{\partial \bar{n}_{ \pm}}{\partial \bar{t}}+\bar{U} \frac{\partial \bar{n}_{ \pm}}{\partial \bar{X}}+\bar{V} \frac{\partial \bar{n}_{ \pm}}{\partial \bar{Y}}\right)=D\left(\frac{\partial^{2} \bar{n}_{ \pm}}{\partial \bar{X}^{2}}+\frac{\partial^{2} \bar{n}_{ \pm}}{\partial \bar{Y}^{2}}\right) \pm \frac{D e z}{T_{e} K_{B}}\left[\frac{\partial}{\partial \bar{X}}\left(\bar{n}_{ \pm} \frac{\partial \bar{\Phi}}{\partial \bar{X}}\right)+\frac{\partial}{\partial \bar{Y}}\left(\bar{n}_{ \pm} \frac{\partial \bar{\Phi}}{\partial \bar{Y}}\right)\right]$

Where $D$ the diffusion coefficient, $K_{B}$ the Boltzmann constant, $T_{e}$ is the average temperature of the electrolytic solution.

And the boundary conditions accompanying the wall are

$\frac{\partial \bar{U}}{\partial \bar{Y}}=0, \bar{T}=T_{0}, \bar{C}=C_{0}, \quad$ at $\quad \bar{Y}=0$

$\bar{U}=0, \bar{T}=T_{1}, \bar{C}=C_{1}$, at $\bar{Y}= \pm \bar{H}(\bar{X}, \bar{t})$

Defining the following quantities to non-dimensionalize the equations governing the flow problem

$x=\frac{\bar{X}}{\lambda}, \quad y=\frac{\bar{Y}}{a}, \quad t=\frac{c \bar{t}}{\lambda}, \quad p=\frac{a^{2} \bar{P}}{c \mu \lambda}, \quad \delta=\frac{a}{\lambda}, \quad u=\frac{\bar{U}}{c}, \quad v=\frac{\bar{V}}{\delta c}, R e=\frac{\rho c a}{\mu}$,

$S_{i j}=\frac{a \bar{S}_{i j}}{c \mu}, \theta=\frac{\bar{T}-T_{0}}{T_{1}-T_{0}}, n=\frac{\bar{n}}{n_{0}}, \zeta=\frac{c^{2} \mu^{2} \bar{\zeta}}{a^{2}}, \varphi=\frac{\bar{\varphi}}{a}, C=\frac{\bar{C}-C_{0}}{C_{1}-C_{0}}, U_{H S}=-\frac{\bar{E}_{\bar{x}} \xi \varepsilon_{0}}{\mu c}$,

$\Phi=\frac{\bar{\Phi}}{\xi}, S_{c}=\frac{\mu}{\rho D}, P_{r}=\frac{\mu c_{p}}{\kappa}, h=\frac{\bar{H}}{a} S=\frac{\sigma \bar{E}_{\bar{X}}^{2} a^{2}}{\kappa\left(T_{1}-T_{0}\right)}, G_{r t}=\frac{\rho g \beta_{T} a^{2}\left(T_{1}-T_{0}\right)}{\mu c}$ 
$G_{r c}=\frac{\rho g \beta_{C} a^{2}\left(C_{1}-C_{0}\right)}{\mu c}, D_{u}=\frac{D K_{T}\left(C_{1}-C_{0}\right)}{c_{\rho} C_{S} \mu\left(T_{1}-T_{0}\right)}, S_{r}=\frac{\rho D K_{T}\left(T_{1}-T_{0}\right)}{\mu T_{m}\left(C_{1}-C_{0}\right)}$

Where $x$ the non-dimensional axial coordinate, $y$ the non-dimensional transverse coordinate, $t$ the non-dimensional time, $p$ the non-dimensional pressure, $\delta$ the wavenumber, $u, v$ the nondimensional axial and transverse velocity components, $R e$ the Reynolds number, $S_{i j}$ the nondimensional shear stress, $\theta$ the non-dimensional temperature, $n$ non-dimensional bulk concentration (number density) of the ions in the electrolyte, $n_{0}$ the concentration of ions at the bulk, $\zeta$ the coefficient of pseudo-plasticity, $C$ the non-dimensional concentration, $U_{H S}$ the Helmholtz-Smoluchowski velocity, $\Phi$ the non-dimensional electric potential, $\xi$ the zeta potential, $S_{c}$ the Schmidt number, $P_{r}$ the Prandtl number, $h$ the non-dimensional transverse wall displacement, $S$ the normalized generation term that represents the ratio of Joule heating to surface heat flux(for constant wall temperature), $G_{r t}$ the local temperature Grashof number, $G_{r c}$ the local concentration Grashof number, $D_{u}$ the Dufour number, $S_{r}$ the Soret number.

After employing Eq.(13) in Eqs. (2)-(7) and (10), with rearrangement, the following forms will be produced.

$\delta\left(\frac{\partial u}{\partial x}+\frac{\partial v}{\partial y}\right)=0$

$\operatorname{Re} \delta\left(\frac{\partial u}{\partial t}+u \frac{\partial u}{\partial x}+v \frac{\partial u}{\partial y}\right)=-\frac{\partial p}{\partial x}+\delta \frac{\partial S_{x x}}{\partial x}+\frac{\partial S_{x y}}{\partial y}-\frac{a^{2}}{\mu c} \frac{\mu c}{\xi \varepsilon_{0}} e z n_{0}\left(n^{+}-n^{-}\right) U_{H S}+G_{r t} \theta+G_{r c} C$

$R e \delta^{3}\left(\frac{\partial v}{\partial t}+u \frac{\partial v}{\partial x}+v \frac{\partial v}{\partial y}\right)=-\frac{\partial p}{\partial y}+\delta^{2} \frac{\partial S_{y x}}{\partial x}+\delta \frac{\partial S_{y y}}{\partial y}$

$R e P_{r} \delta\left(\frac{\partial \theta}{\partial t}+u \frac{\partial \theta}{\partial x}+v \frac{\partial \theta}{\partial y}\right)=\delta^{2} \frac{\partial^{2} \theta}{\partial x^{2}}+\frac{\partial^{2} \theta}{\partial y^{2}}+\frac{D_{m} K_{T}\left(C_{1}-C_{0}\right)}{C_{s} \kappa\left(T_{1}-T_{0}\right)} \delta^{2} \frac{\partial^{2} C}{\partial x^{2}}+\frac{D_{m} K_{T}\left(C_{1}-C_{0}\right)}{C_{S} \kappa\left(T_{1}-T_{0}\right)} \frac{\partial^{2} C}{\partial y^{2}}+\frac{a^{2}}{\kappa\left(T_{1}-T_{0}\right)} \sigma \bar{E}_{\bar{X}}^{2}$

$\operatorname{Re} S_{c} \delta\left(\frac{\partial C}{\partial t}+u \frac{\partial C}{\partial x}+v \frac{\partial C}{\partial y}\right)=\delta^{2} \frac{\partial^{2} C}{\partial x^{2}}+\frac{\partial^{2} C}{\partial y^{2}}+\frac{K_{T}\left(T_{1}-T_{0}\right)}{T_{m}\left(C_{1}-C_{0}\right)} \delta^{2} \frac{\partial^{2} \theta}{\partial x^{2}}+\frac{K_{T}\left(T_{1}-T_{0}\right)}{T_{m}\left(C_{1}-C_{0}\right)} \frac{\partial^{2} \theta}{\partial y^{2}}$

$\operatorname{Re} S_{C} \delta\left(\frac{\partial n_{ \pm}}{\partial t}+u \frac{\partial n_{ \pm}}{\partial x}+v \frac{\partial n_{ \pm}}{\partial y}\right)=\delta^{2} \frac{\partial^{2} n_{ \pm}}{\partial x^{2}}+\frac{\partial^{2} n_{ \pm}}{\partial y^{2}} \pm \delta^{2} \frac{e z}{T_{e} K_{B}} \frac{\partial}{\partial x}\left(n_{ \pm} \frac{\partial \xi \Phi}{\partial \lambda x}\right)+\frac{e z \xi}{T_{e} K_{B}} \frac{\partial}{\partial y}\left(n_{ \pm} \frac{\partial \Phi}{\partial y}\right)$

Applying the lubrication theory, as is customary for peristaltic hydrodynamics, the following emerging linearized conservation equations are obtained

$-\frac{\partial p}{\partial x}+\frac{\partial S_{x y}}{\partial y}-\frac{a^{2}}{\mu c} \frac{\mu c}{\xi \varepsilon_{0}} e z n_{0}\left(n^{+}-n^{-}\right) U_{H S}+G_{r t} \theta+G_{r c} C=0$

$\frac{\partial p}{\partial y}=0$

$\frac{\partial^{2} \theta}{\partial y^{2}}+P_{r} D_{u} \frac{\partial^{2} C}{\partial y^{2}}+S=0$

$\frac{\partial^{2} C}{\partial y^{2}}+S_{C} S_{r} \frac{\partial^{2} \theta}{\partial y^{2}}=0$

And the non-dimensional constituent equation for the Rabinowitsch fluid model is

$S_{x y}+\zeta S_{x y}^{3}=\frac{\partial u}{\partial y}$

The non-dimensional associated boundary conditions are

$\frac{\partial u}{\partial y}=0, \theta=0, C=0, S_{x y}=0 \quad$ at $\quad y=0$

$u=0, \theta=1, C=1, \quad$ at $\quad y= \pm h(x, t)$

And the non-dimensional Nernst-Planck equation becomes

$\frac{\partial^{2} n_{ \pm}}{\partial y^{2}}+\frac{e z \xi}{T_{e} K_{B}} \frac{\partial}{\partial y}\left(n_{ \pm} \frac{\partial \Phi}{\partial y}\right)=0$ 
It is necessary to calculate the charge number density to obtain the value of the potential distribution and this is done by finding the solution of equation. (27) with help the bulk conditions $n_{ \pm}=1$ at $\Phi=0$ and $\frac{\partial n}{\partial y}=0$ at $\frac{\partial \Phi}{\partial y}=0$, and the result is

$n_{ \pm}=e^{\mp \frac{e z \xi}{T_{e} K_{B}} \Phi}$

By substituting (28) in equation.(20), we obtain the electrical distribution as follows, we obtain the electrical distribution as follows

$\frac{\partial^{2} \Phi}{\partial y^{2}}=-\frac{a^{2} e z n_{0}}{\xi \varepsilon_{0}}\left(e^{+\frac{e z \xi}{T_{e} K_{B}} \Phi}-e^{-\frac{e z \xi}{T_{e} K_{B}} \Phi}\right)$

We can write in another form as

$\frac{\partial^{2} \Phi}{\partial y^{2}}=-\frac{a^{2} e z n_{0}}{\xi \varepsilon_{0}} \sinh \left(\frac{e z \xi}{T_{e} K_{B}} \Phi\right)$

Now by employing the Debye-Hückel linearization approximation the form of the Poisson-Boltzmann equation is transformed as follows

$\frac{\partial^{2} \Phi}{\partial y^{2}}=-m^{2} \Phi$

Where $m=$ a e $z \sqrt{\frac{2 n_{0}}{\varepsilon_{0} T_{e} K_{B}}}=\frac{a}{\lambda_{d}}$ and is called the electro-osmotic parameter, $\lambda_{d}$ is Debye length or characteristic thickness of the electrical double layer (EDL).

By solving the equation.(31) which subject to boundary conditions

$\left.\frac{\partial \Phi}{\partial y}\right|_{y=0}=0$ and $\left.\Phi\right|_{y=h}=1$, the potential distribution function is found as follows

$\Phi=\frac{\cosh (m y)}{\cosh (m h)}$

Now Eq.(20) can be written in the following form

$-\frac{\partial p}{\partial x}+\frac{\partial S_{x y}}{\partial y}+m^{2} \Phi U_{H S}+G_{r t} \theta+G_{r c} C=0$

\section{Analytical solutions}

The temperature and concentration equations are obtained when solving equation. (22) and equation.(23) simultaneous using the boundary conditions equation (25)and (26)as follows

$\theta=\frac{-2 y-h^{2} y S+h y^{2} S+2 y D_{u} P_{r} S_{c} S_{r}}{2 h\left(-1+D_{u} P_{r} S_{c} S_{r}\right)}$

$C=\frac{-2 y+h^{2} y S S_{c} S_{r}-h y^{2} S S_{c} S_{r}+2 y D_{u} P_{r} S_{c} S_{r}}{2 h\left(-1+D_{u} P_{r} S_{c} S_{r}\right)}$

Substitute equation.(34) and equation (35) in equation (33) and integrating it with using the boundary conditions equation.(25), the following result yield

$S_{x y}=\frac{1}{12 h\left(-1+D_{u} P_{r} S_{c} S_{r}\right)}\left(-12 h \frac{\partial p}{\partial x} y+6 y^{2} G_{r c}+6 y^{2} G_{r t}+3 h^{2} y^{2} S G_{r t}-2 h y^{3} S G_{r t}-\right.$ $3 h^{2} y^{2} S G_{r c} S_{c} S_{r}+2 h y^{3} S G_{r c} S_{c} S_{r}+\ldots$

(36)

The axial velocity is found by compensation equation (36) in (24) and using the remaining conditions, the $u$ is as follows

$u=\left(362880 h^{4} m^{6} \frac{\partial p}{\partial x} \operatorname{Cosh}[h m]+181440 h^{6} m^{6} \frac{\partial p^{3}}{\partial x} \zeta \operatorname{Cosh}[h m]+120960 h^{4} m^{6} \frac{\partial p}{\partial x} \operatorname{Cosh}[3 h m]+\right.$ $60480 h^{6} m^{6} \frac{\partial p^{3}}{\partial x} \zeta \operatorname{Cosh}[3 h m]-120960 h^{4} m^{6} \operatorname{Cosh}[h m] G_{r c}-$

$217728 h^{6} m^{6} \frac{\partial p^{2}}{\partial x} \zeta \operatorname{Cosh}[h m] G_{r c}-\ldots$

The volumetric flow rate can be defined as follows

$Q=\int_{0}^{h} u d y$ 
The relationship between the laboratory frame and the wave frame can be represented by the following transformation

$\bar{x}=x-t, \quad \bar{y}=y, \quad \bar{u}=u-c, \quad \bar{v}=v$,

It can express the volumetric flow rate in the wave frame as follows

$\bar{q}=\int_{0}^{h(\bar{x})} \bar{u}(\bar{x}, \bar{y}) d \bar{y}=\int_{0}^{h(x)}(u-1) d \bar{y}$

From equations (38) -(40) yield

$\bar{q}=Q-h$

Averaging volumetric flow rate over one time period, we obtain

$Q^{*}=\int_{0}^{1} Q d t=\int_{0}^{1}(\bar{q}+h) d t=\bar{q}+1-\frac{\varphi}{2}$

Rewrite equation (55) as

$Q^{*}=Q-h+1-\frac{\varphi}{2}$

The dimensionless form of the volumetric flow rate in the laboratory frame

by virtue of equation (38), is

$Q=\left(362880 h^{4} m^{6} \frac{\partial p}{\partial x} \operatorname{Cosh}[h m]+181440 h^{6} m^{6} \frac{\partial p^{3}}{\partial x} \zeta \operatorname{Cosh}[h m]+120960 h^{4} m^{6} \frac{\partial p}{\partial x} \operatorname{Cosh}[3 h m]+\right.$ $60480 h^{6} m^{6} \frac{\partial p^{3}}{\partial x} \zeta \operatorname{Cosh}[3 h m]-120960 h^{4} m^{6} \operatorname{Cosh}[h m] G_{r c}-217728 h^{6} m^{6} \frac{\partial p^{2}}{\partial x} \zeta \operatorname{Cosh}[h m] G_{r c}-$

$40320 h^{4} m^{6} \operatorname{Cosh}[3 h m] G_{c}-72576 h^{6} m^{6} \frac{\partial p^{2}}{\partial x} \zeta \operatorname{Cosh}[3 h m] G_{r c}+\cdots$

From the above equation, we can derive the pressure gradient by integrating Eq. (44)

$\frac{\partial p}{\partial x}=\left(5\left(-42 h^{5} m^{3} \zeta \operatorname{Cosh}[h m] G_{r c}-14 h^{5} m^{3} \zeta \operatorname{Cosh}[3 h m] G_{r c}-42 h^{5} m^{3}+\zeta \operatorname{Cosh}[h m] G_{r t}\right.\right.$

$-9 h^{7} m^{3} \zeta S \operatorname{Cosh}[h m] G_{r t}-14 h^{5} m^{3} \zeta \operatorname{Cosh}[3 h m] G_{r t}-3 h^{7} m^{3} \zeta S \operatorname{Cosh}[3 h m] G_{r t}$

$+9 h^{7} m^{3} \zeta S \operatorname{Cosh}[h m] G_{r c} S_{c} S_{r}+3 h^{7} m^{3} \zeta S \operatorname{Cosh}[3 h m] G_{r c} S_{c} S_{r}+42 h^{5} m^{3} \zeta \operatorname{Cosh}[h m] D_{u} G_{r c} P_{r} S_{c} S_{r}+$

$14 h^{5} m^{3} \zeta \operatorname{Cosh}[3 h m] D_{u} G_{r c} P_{r} S_{c} S_{r}+42 h^{5} m^{3} \zeta \operatorname{Cosh}[h m] D_{u} G_{r t} P_{r} S_{c} S_{r}+\cdots$

The pressure difference across one wavelength is calculated from the previous equation

$\Delta p=\int_{0}^{1} \frac{\partial p}{\partial x} d x$

The stream function is related to the velocity components in the wave frame by the following formula after resorting to equation (37)

$\bar{u}=\frac{\partial \psi}{\partial \bar{y}}, \bar{v}=-\frac{\partial \psi}{\partial \bar{x}}$

The heat transfer coefficient at the wall is defined by

$\mathrm{Z}(x)=h_{x} \theta_{y}(h)$

\section{Numerical results and discussion}

The main purpose of this section is to monitor the physical behavior of the Rabinowitsch fluid model flow as it passes through a very small channel and this behavior is embodied by graphs and how the parameters are associated with the problem affect the flow. Mathematica program is used to visualize flow behavior.

\subsection{Velocity Profile}

The velocity profile response to variation in pertinent parameters as external electric field $U_{\mathrm{HS}}$, Joule heating $S$, the Dufour number $D_{u}$, the Soret number $S_{r}$ and the coefficient of Rabinowitsch fluid $\zeta$ is depicted in Figures (2)(a-k). The snapshots of the axial velocity exhibit asymmetric and skewed shape about the midline of the channel also are observed deviations of the axial velocity from the foreseeable parabolic shape. Figure (a) shows the impact of the external electric field which can be simulated by maximum electro osmotic 
velocity or Helmholtz-Smoluchowski $U_{H S}=-\frac{E_{x} \xi \varepsilon_{0}}{\mu c}$ on the axial velocity. It is clear from the diagram when the value of electric field high leads to boost the Helmholtz-Smoluchowski, in turn to superfat the electro-kinetic body force which is assistive to acceleration flux fluid in the center of the channel, while it is inhibitive whenever to get closer to the channel wall. Obviously, the summit of the parabola velocity distribution increases and become more consistent with an increase $U_{H S}$ which means an increase in the external electric field. Among the benefits of the electric field is the acceleration of the flow in the electric kinetics, as it is found that it is very sensitive to the photoelectric flow, which leads to relatively low changes in the intensity of the electric field. This nature is considered a benefit in practical, analog, micro-electric pumps, as very good acceleration in the basic flow that can be accomplished with relatively slight adjustments in the electric field. This, in turn, reduces costs at the same time and addresses the problems of electromagnetic compatibility. Figure (b) illustrates the role of Joule heating on the axial velocity. It is clear that the velocity accelerate toward the charge carrying walls with a significant drop off towards the center of the conduit when increasing the positive value $S$ with $U_{\mathrm{HS}}=1$ and vice versa, a slowdown in the axial velocity occur when increasing negative values $S$. A concentration gradient of positive ions is created near the wall surface due to the existence of negative ion charges on the wall surface and as a result. There is an electrical potential distribution in the electrolyte which is called the electric double layer. The height of the (axial) electric field leads to a quadratically increase in the Joule parameter(for constant temperature difference) also the electro-kinetic body force significantly boosts, in Outcome, there will be an acceleration in the axial flux when Joule heating is positive, While deceleration occurs in flux when Joule heating is negative. It is evident that the Joule parameter incites a significant modification in flux distribution through the channel and confirms the stellar ability of electro-osmotic phenomena in controlling velocity profiles at the microchannel walls. Figure (c)-(d) depict the variation of the axial velocity against the Soret number $S_{r}$ for two different value to $U_{\mathrm{HS}}$, where one can see from Figure (c) that increasing the Soret number with $U_{\mathrm{HS}}=1$, causes inhibitive axial velocity on the left side of the channel, while the effect is almost non-existent on the other side. When $U_{\mathrm{HS}}$ is negative the maximum velocity emerges at the conduit walls while the velocity is minimum at the core region that is clear in Figure (d). Figure (e) reveals the impact of the Dufour number $D_{u}$ on the velocity profile. The increase in value $D_{u}$ leads to enhance the axial velocity in the region near the wall with $U_{\mathrm{HS}}=1$. The velocity accelerates at the center core zone of the conduit with increasing $D_{u}$ with case the $U_{\mathrm{HS}}=-1$ see Figure (f). The last diagram Figure $(\mathrm{g})$ shows the effect of the coefficient of Rabinowitsch fluid $\zeta$ on the axial velocity. The effect of the parameter is limited to the sides of the channel. It reduces the axial velocity in this region, while it has almost no effect in the core region. 


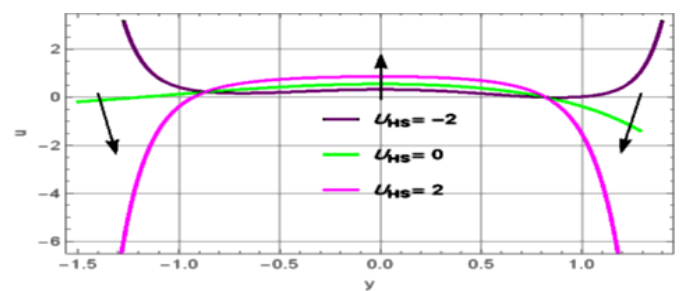

(a)

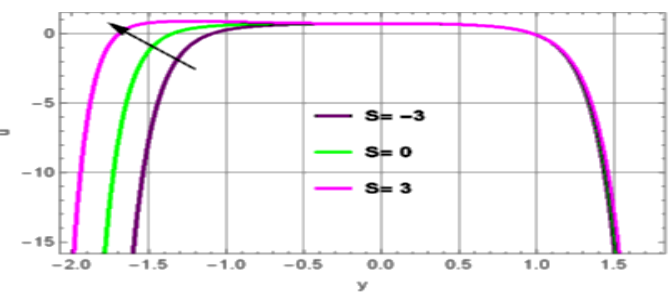

(b)

Figure2.the axial velocity profile for variation of (a): the Helmholtz-Smoluchowski velocity $U_{H S}$, (b): the Joule heating $S$ with $\left(U_{\mathrm{HS}}=1\right)$, and for fixed values of parameters $\left(Q^{*}=0.5, \varphi=0.2, \zeta=0.2, D_{u}=0.6, P_{r}=\right.$ $\left.0.2, S_{c}=0.5, S_{r}=0.3, m=2, G_{r c}=0.5, G_{r t}=0.3, x=0.1, t=0.1\right)$.

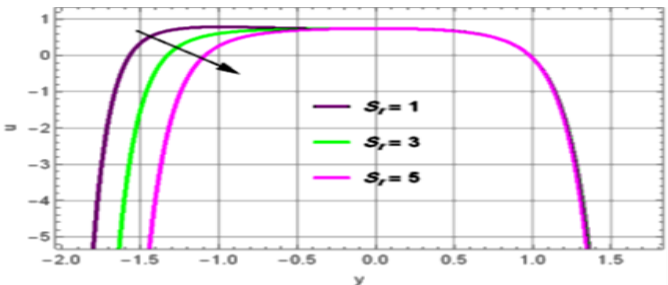

(c)

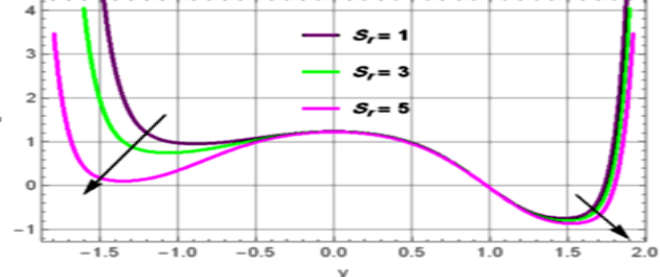

(d)

Figure2.the axial velocity profile for variation of, (c): the Soret number $S_{r},\left(U_{\mathrm{HS}}=1\right)(\mathbf{d})$ : the Soret number $S_{r},\left(U_{\mathrm{HS}}=-1\right)$, and for fixed values of parameters $\left(Q^{*}=0.5, \varphi=0.2, \zeta=0.5, m=2, D_{u}=0.6, P_{r}=\right.$ $0.2, S_{c}=0.5, S_{r}=1, S=2, G_{r c}=0.5, G_{r t}=0.7, x=0.5, t=0.1$ )

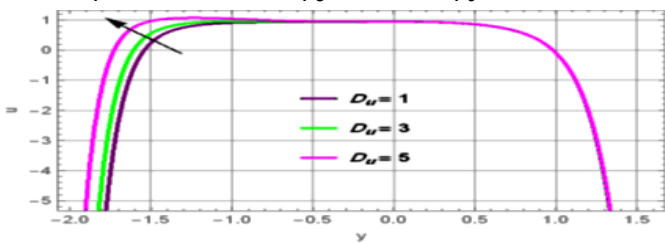

(e)

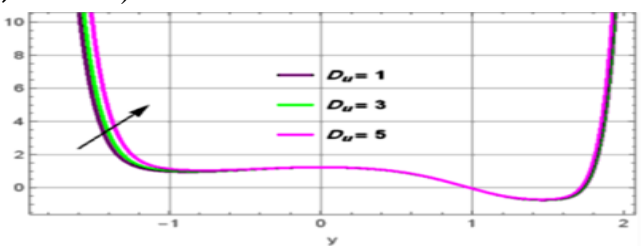

(f)

Figure2. the axial velocity profile for variation of , (e): the Dufour number $D_{u}\left(U_{\mathrm{HS}}=1\right)$, (f): the Dufour number $D_{u}\left(U_{\mathrm{HS}}=-1\right)$, and for fixed values of parameters $\left(Q^{*}=0.5, \varphi=0.2, \zeta=0.5, m=2, D_{u}=0.6\right.$, $\left.P_{r}=0.2, S_{c}=0.5, S_{r}=1, S=2, U_{\mathrm{HS}}=1, G_{r c}=0.5, G_{r t}=0.7, x=0.5, t=0.1\right)$

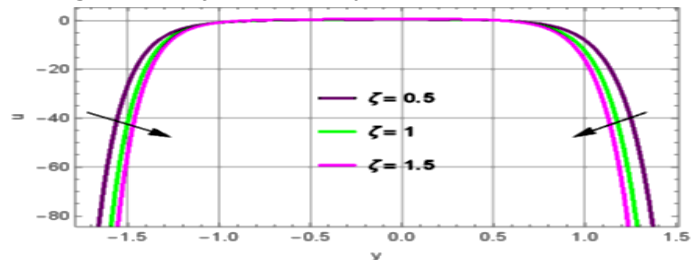

(g)

Figure2. the axial velocity profile for variation of, $(\mathrm{g})$ : the coefficient of Rabinowitsch fluid, and for fixed values of parameters $\left(Q^{*}=0.5, \varphi=0.5, m=2, D_{u}=0.6, P_{r}=1.2, S_{c}=0.5, S_{r}=0.5, S=2, U_{\mathrm{HS}}=\right.$ $\left.1, G_{r c}=0.5, G_{r t}=0.7, x=0.2, t=0.1\right)$.

\subsection{Temperature Distribution}

The parameters effect on the temperature profile is analyzed via Figures (3)(a-c) and from the first look at the graphs, it is noted that with the positive values of Joule heating, the temperature decreases and is concentrated in the middle area of the channel and its values are in the negative part of the axis of $y$. Where Figure (a) reveals the impact of the positive and negative values of Joule heating $S$ on the distribution of temperature. It is observed that the highest temperature between $y \in[0,1]$ when $S$ is positive but the heat is progressively decreases until its values are negative which is means induces refrigeration in the electrolyte. The influence will be reversed with the negative value of $S$ where the temperature increases and its value becomes positive. It is also observed that in the absence of the influence of Joule 
heating $S=0$, the temperature profile would be linear. It can be observed from Figure (b) that the Dufour number $D_{u}$ has a negative effect on the temperature profile since a decrease in temperature is observed in the areas near the wall. Figure (c) shows the receding of temperature toward the core area, with increasing of the Soret number $S_{r}$ which makes the sides cooler than the middle.

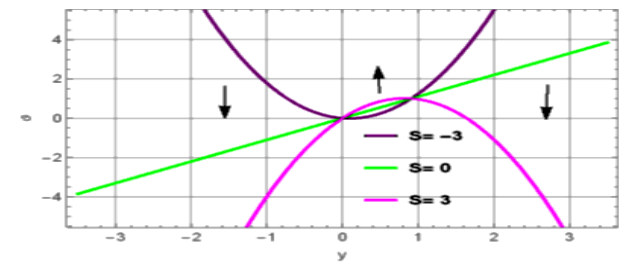

(a)

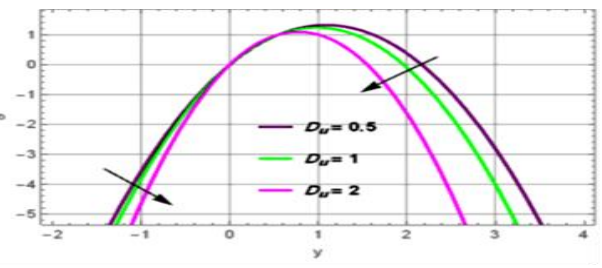

(b)

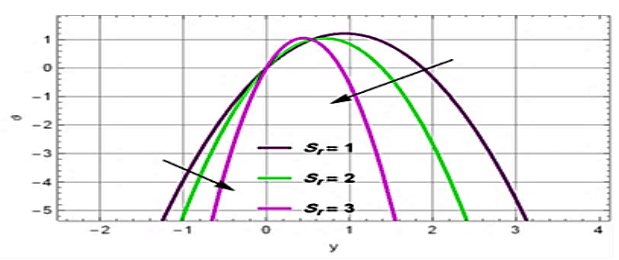

( c)

Figure3. the temperature distribution for variation of, (a): the Joule heating $S$,(b): the Dufour number $D_{u}$, (c): the Soret number $S_{r}$, and for fixed values of parameters $\left(\varphi=0.5, P_{r}=1.5, S_{c}=0.3, x=0.2, t=0.1\right.$ ).

\subsection{Concentration profile}

The concentration is affected by the diversity of the governing parameters, and this is shown in illustrations Figures (4) (a-c). It is observed that the joule heating $S$ clearly affect the concentration when its values differed between positive and negative. We note from Figure (a) with the positive values of joule heating that the concentration increases gradually and has positively parabolic. While negative values of $S$ show an opposite effect, where the concentration distribution takes negatively parabolic shape. In the absence of joule heating, the concentration is linear. The impact of the Dufour number $D_{u}$ is illustrated in Figure (b) which tells us when the value of number getting more, the concentration increases and the speed of response to the change in the left side of the channel is more than on the right side. Figure (c) presents the influence of the Soret number $S_{r}$ on the concentration, the graph indicates that $S_{r}$ enhance concentration.

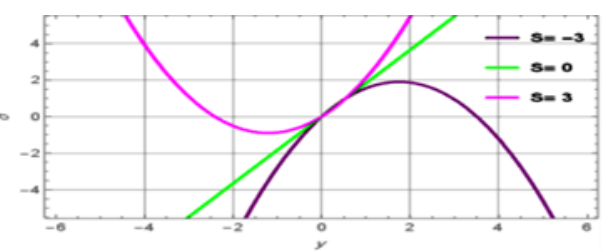

(a)

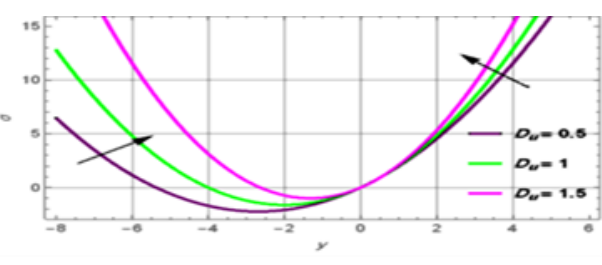

(b) 


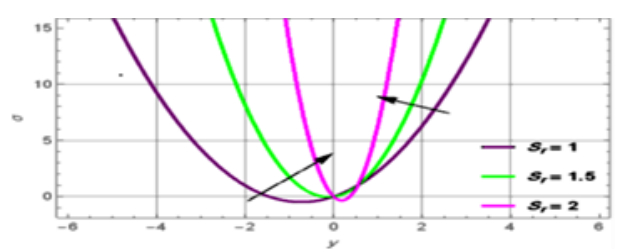

( c)

Figure4. Concentration profile for variation of, (a): the Joule heating $S$, (b): the Dufour number $D_{u}$, (c): the Soret number $S_{r}$, and for fixed values of parameters $\left(\varphi=0.5, P_{r}=1.5, S_{c}=0.5, x=0.2, t=0.1\right)$.

\subsection{Heat Transfer Coefficient}

In this part of the analysis, the reaction of Heat transfer $\mathrm{Z}(x)$ with the diversity of different interesting parameters is recorded. The first vision to the graphs shows the wavy behavior to Heat transfer rate, and this is returned to the relaxing movement and contraction resulted from the peristaltic flow. Figures (5)(a-c) reveals the Heat transfer profile vs. axial coordinate. The behavior of heat transfer suffers fluctuations in the reaction, as the area $(0.1 \leq x \leq 0.6)$ witnessed a decrease in the rate and an increase at $(0.6 \leq x \leq 1.1)$. The presence Dufour number has a slight effect on the Heat transfer rate and this is clear in Figure (a). The influence of Soret number on the Heat transfer rate is indicated in Figure (b). It is shown that the wave shape is more consistent with an increase $S_{r}$. while the behavior reverses with Joule heating the wave be more harmonious with decreases the value of $\mathrm{S}$. This is illustrated in Figure (c).

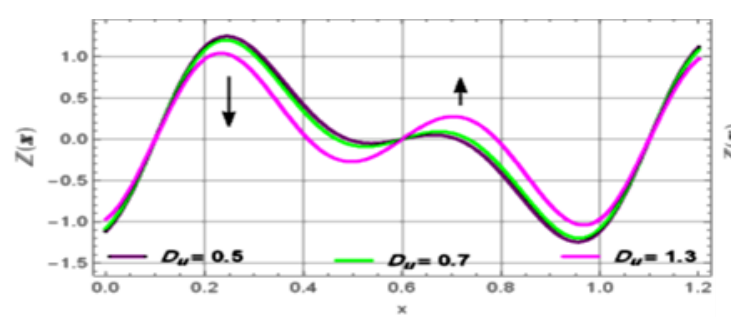

( a)

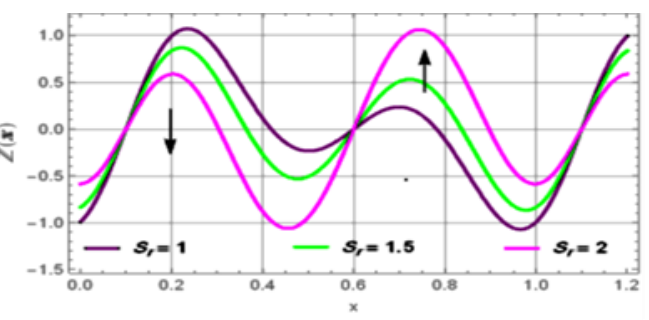

(b)

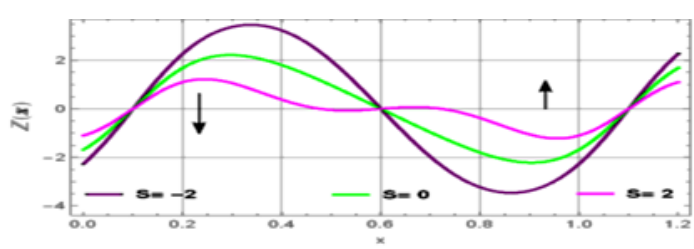

(c)

Figure5. the Heat Transfer Coefficient for variation of (a): the Dufour number $D_{u}$, (b): the Soret number $S_{r}$, (c): the Joule heating $S$, and for fixed values of parameters $\left(\varphi=0.5, P_{r}=1.5, S_{c}=0.3, t=0.1\right)$.

\subsection{Pressure rise}

The complicated integral that appears in equation (46) has been processed numerically with the help of Mathematica to obtain pressure rise $\Delta p$. Figures (6)(a-d) illustrate the behavior of the pressure rise across one wavelength against the averaged volumetric flow rate 
for the Helmholtz-Smoluchowski velocity $U_{H S}$, the Joule heating $S$, the electro-osmotic parameter $m$, and $\zeta$ the coefficient of pseudo-plasticity. There are three regimes of pumping that can be categorized. The positive pumping or the pumping region $(\Delta p>0)$ which considers the peristaltic pumping, the second regime namely the co-pumping region or augmented pumping region $(\Delta p<0)$ and the last the free pumping region. In general, we notice from the drawings that the highest value of pressure rise is in the pumping region with a negative the averaged volumetric flow rate. It is evident that the relation between the pressure rise and the averaged volumetric flow rate is an inverse relationship, the flow rate increases with the decrease in the pressure rise. Figure (a) shows the presence of the applied electric field on the pressure rate. The positive value of $U_{H S}$ obviously, enhances the pumping rate $(\Delta p)$ in the peristaltic pumping and augmented pumping region, while the negative values of $U_{H S}$ depresses the pressure. In the free region we notice that the flow rate takes the highest value when $U_{H S}>0$, but in the absence of the external electric field, the flow rate becomes zero. The influence of Joule heating has been illustrated in Figure (b). Poor improvement in the pumping rate in the peristaltic pumping is induced by raising the magnitude of the Joule heating $S$. Decrease the thickness of the electrical double layer EDL i.e. increasing $m$ (which has an inverse relationship with $\lambda_{d}$ ), lead to increase in the pressure rate $\Delta p$, this is clear in Figure (c). It can be noticed from Figure (d) that the increment in the coefficient of Rabinowitsch fluid, the pumping rate decreases in the peristaltic pumping $\left(\Delta p>0,-2 \leq Q^{*} \leq-0.5\right)$, whereas the attitude of pumping rate changes in $\left(\Delta p>0, Q^{*}>\right.$ $0)$, the pressure rise increases.

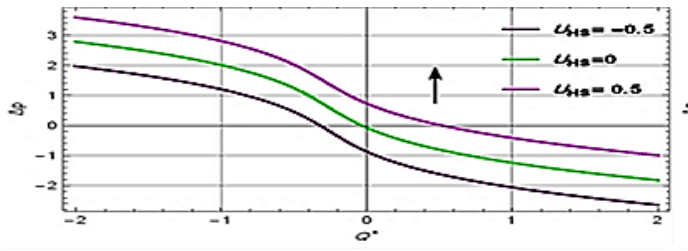

(a)

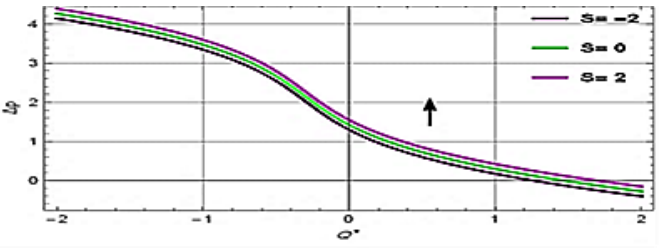

(b)

Figure6. Pressure rise upon the mean flow rate $Q^{*}$ for variation of (a): the Helmholtz-Smoluchowski velocity $U_{H S}$, (b): Joule heating $S$, and for fixed values of parameters $\left(\varphi=0.5, \zeta=0.5, m=2, D_{u}=\right.$ $\left.0.6, P_{r}=1.2, S_{c}=0.3, S_{r}=0.5, G_{r c}=0.5, G_{r t}=0.7, t=0.5\right)$.

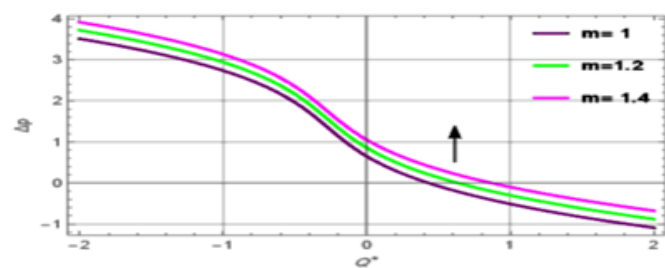

(c)

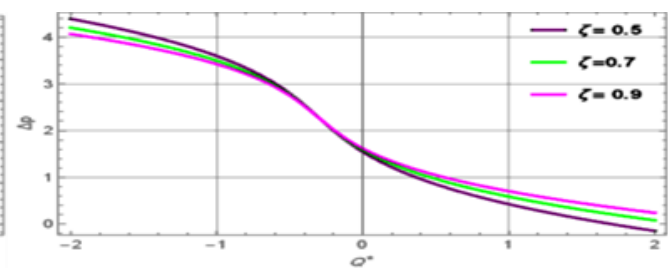

(d)

Figure6. Pressure rise upon the mean flow rate $Q^{*}$ for variation of (c): electro-osmotic parameter $m$, (d): the parameter of Pseudoplastic fluid $\zeta$, and for fixed values of parameters $\left(\zeta=0.5, D_{u}=0.6, P_{r}=1.2, S_{c}=\right.$ $\left.0.3, S_{r}=0.5, S=2, U_{\mathrm{HS}}=1, G_{r c}=0.5, G_{r t}=0.7, t=0.5\right)$.

\subsection{Trapping formulation}

Streamlines visualizations in the conduit for various values of pertinent parameters for a fixed time $(t=0.1)$ is illustrated in Figures (7) (a-d), and this visualization lets a better examination of the so-called trapping phenomenon, wherein an internally circulating bolus of 
the fluid is formed by closed streamlines. In all the graphs, there is a considerable shortage of symmetry about the midline $(y=0)$. In a particular, evident distortion of streamlines emerges in Figure (a) when the magnitudes of the external electric field or (the HelmholtzSmoluchowski velocity $U_{H S}$ ) differ between positive and negative values. At $U_{H S}=-1$, the numbers of trapping bolus increase at high transverse coordinate value, whereas, in the lower coordinates, the streamlines testify a shift from the bolus to the curves. Figure (b) displays the effect of the Dufour number $D_{u}$ on streamline. It is revealed that the number of the trapping bolus decreases for a larger value of $D_{u}$. The impact of the Soret number $S_{r}$ on the bolus is sketched in Figure (c). It is evident from the diagram that ascending values of $S_{r}$ lead to an increase in the number and size of the bolus. It is revealed in Figure (d) that the number of trapping bolus increases in the upper zone of the channel, whereas the bolus begins gradually shrinking in the number and expanding in the size at a lower region with $S<0$.
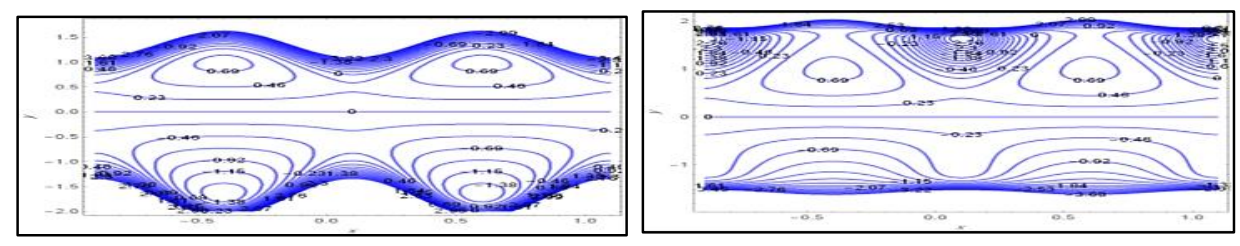

(a)

Figure7. Streamlines for variation of the Helmholtz-Smoluchowski velocity $U_{H S}$, (a): $U_{H S}=1, U_{H S}=-1$ and fixed $\left(Q^{*}=0.5, \varphi=0.5, \zeta=0.5, m=2, D_{u}=0.6, P_{r}=1.2, S_{c}=0.5, S_{r}=0.3, S=2, G_{r c}=1.5, G_{r t}=2.5\right)$
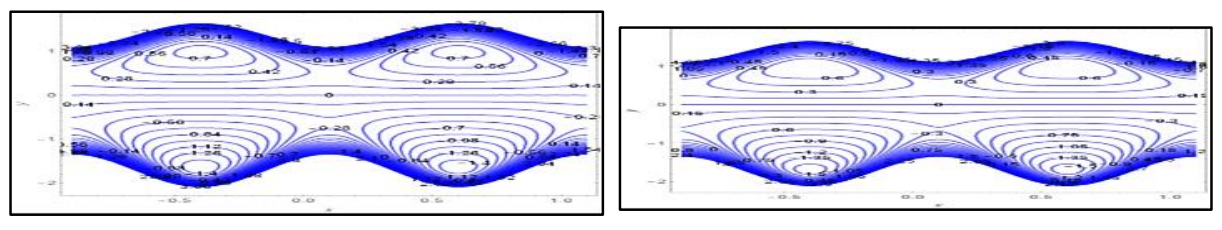

(b)

Figure7. Streamlines for variation of the Dufour number $D_{u}$, (b): $D_{u}=0.4, D_{u}=1$ and fixed $\left(Q^{*}=0.5, \varphi=\right.$ $0.5, \zeta=0.5, m=2, P_{r}=1.2, S_{c}=0.5, S_{r}=0.3, S=2, U_{\mathrm{HS}}=1, G_{r c}=1.5, G_{r t}=2.5$ )
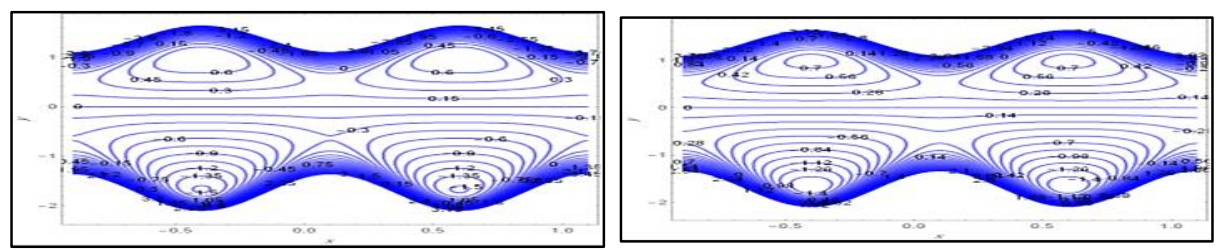

(c)

Figure7. Streamlines for variation of the Soret number $S_{r}$, (c): $S_{r}=0.5, S_{r}=1$ and fixed $\left(Q^{*}=0.5, \varphi=\right.$ $\left.0.5, \zeta=0.5, m=2, D_{u}=0.6, P_{r}=1.2, S_{c}=0.5, S=2, U_{\mathrm{HS}}=1, G_{r c}=1.5, G_{r t}=2.5\right)$.
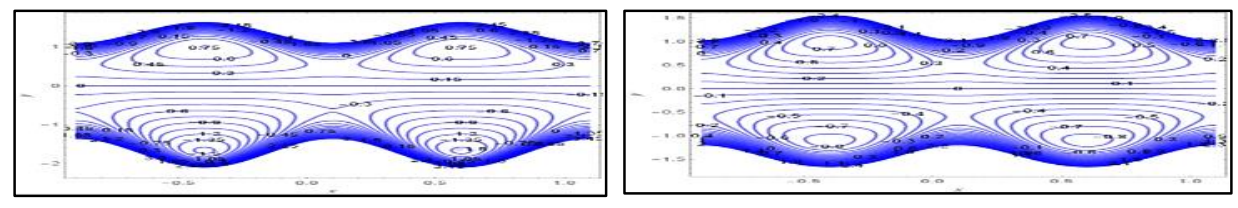

(d)

Figure7. Streamlines for variation of the Joule heating $S,(\mathbf{d}): S=2, S=-2$ and fixed $\left(Q^{*}=0.5, \varphi=0.5, \zeta=\right.$ $0.5, m=2, D_{u}=0.6, P_{r}=1.3, S_{c}=0.5, S_{r}=0.3, \quad U_{\mathrm{HS}}=1, G_{r c}=1.5, G_{r t}=2.5$ ) 


\section{Conclusion}

The mathematical and theoretical studies on the unsteady electro-osmotic peristaltic pumping in an isothermal finite length micro-channel have been conducted to investigate the effect of Soret and Dufour phenomenon and Joule electrothermal heating on Rabinowitsch fluid model. Mathematica program has been employed to evaluate numerical solutions derived for the axial velocity, the temperature profile, concentration, the heat transfer coefficient, and the pressure rise after simplified the electrokinetic transport equations via the lubrication theory. Key observations are

1- The axial velocity is increasing functions of the external electric field $U_{\mathrm{HS}}$ and this result agrees with offered in Tripathi et.al. $[8,10]$ while the opposite behavior is presented in Jayavel [14].

2- The axial velocity shows flat parabolic behavior in nature. This behavior increases at a left wall via the positive Joule heating $S$, the Dufour number $D_{u}$. While decreasing behavior is observed due to the Soret number $S_{r}$.the negative Joule heating $S$ accelerates the axial velocity in the center of the channel whereas inhibited at the wall.

3- With increasing $D_{u}$ and $S_{r}$, the temperature distribution drops and the positive joule heating raises the heat in mid of the channel while it slows down the heat near the wall. With the negative joule heating, the temperature takes exactly reversal behavior.

4- The concentration field is an increasing function of the applied electric field (which represented by Joule heating $\left.S=\frac{\sigma \bar{E}_{\bar{X}}^{2} a^{2}}{\kappa\left(T_{1}-T_{0}\right)}\right), D_{u}$ and $S_{r}$.

5- The pressure rise increases with $S$, electro-osmosis parameter $m$, whereas the pressure rise testifies a changeful behavior with $\zeta$.

6- The trapping bolus distort with negative the external electric field $U_{\mathrm{HS}}$.

7- The trapping bolus expands with increasing $S_{r}$, and $S<0$, whilst the number of boluses decreases with $D_{u}$ 


\section{References}

1. Hasen, S. S.; Abdulhadi, Ah. M., Influence of a Rotating Frame on the Peristaltic Flow of a Rabinowitsch Fluid Model in an Inclined Channel, Journal of Al-Qadisiyah for Computer Science and Mathematics, 2020,12, 1, 21-33.

2.Kotnurka, As. Sh.; Giddaiah, S., Bioconvection peristaltic flow of nano Eyring-Powell fluid containing gyrotactic microorganism, SN Applied Sciences, 2019 1:1276.

3.Kareem, R. S; Abdul-Hadi Ah. M., The effect of MHD and Porous Media on Nanofluid Flow with Heat Transfer: Numerical Treatment, Journal of advanced research in fluid mechanics and thermal sciences, 2019,63 issues (2),317-328.

4. Ali, H. A.; Abdulhadi, Ah. M., the Peristaltic Transport of MHD Eyring- Powell Fluid Through Porous Medium in a Three Dimensional Rectangular duct, International Journal of Pure and Applied Mathematics, 2018,119,18.

5. Salman, M. R.; Abdulhadi, Ah. M., Analysis of Heat and Mass Transfer in a Tapered ASymmetric Channel During Peristaltic Transport of (Pseudo plastic Nano Fluid) with variable Viscosity Under the Effect of (MHD), Journal of al-Qadisiyah for Computer Science and Mathematics, 2018,10,3,80-96.

6.Chakraborty, S., Augmentation of peristaltic microflows through electro-osmotic mechanisms, J. Phys. D: Appl. Phys. 2006,39 , 5356-5363.

7.Tripathi, Dh.; Mulchandani, J.; Jhalani, Sh. , Electrokinetic transport in unsteady flow through peristaltic microchannel, AIP Conf. Proc, the American Institute of Physics, 2016, 1724, 020043 .

8.Tripathi, Dh.; Sharma, A.; Bég, O. A., Joule heating and buoyancy effects in electroosmotic peristaltic transport of aqueous nanofluids through a microchannel with complex wave propagation, Advanced Powder Technology, APT 1812, 2017,15.

9.Tripathi, Dh.; Sharma, A.; Bég, O. A., Electrothermal transport of nanofluids via peristaltic pumping in a finite micro-channel: Effects of Joule heating and Helmholtz-Smoluchowski velocity, International Journal of Heat and Mass Transfer, 2017,111,138-149.

10. Tripathi, Dh.; Bhushan, Sh.; Bég, O. A., Unsteady viscous flow driven by the combined effects of peristalsis and electro-osmosis, Alexandria Engineering Journal ,2018, 57, 13491359.

11.Tripathi, Dh.; Yadav, A.; Bég, O. A.r, Electro-Kinetically Driven Peristaltic Transport Of Viscoelastic Physiological Fluids Through A Finite Length Capillary: Mathematical Modelling, Mathematical Biosciences, 2017,283, 155-168.

12.Tripathi, Dh.; Jhorar, R.; Bég, O. A., Shaw. S., Electroosmosis modulated peristaltic biorheological flow through an asymmetric microchannel: mathematical model, Springer,2017.

13.Tripathi, Dh.; Sharma. A.; Bég. O. A; Tiwari. Ab., Electrothermal Transport in Biological Systems: An Analytical Approach for Electrokinetically-Modulated Peristaltic Flow, J. Thermal Sci. Eng. Appl. , 2017,9,4: 041010 (9 pages).

14. Jayavel, P.; Jhorar, R.; Tripathi, Dh.; Azese, M. N., Electroosmotic flow of pseudoplastic nanoliquids via peristaltic pumping, Journal of the Brazilian Society of Mechanical Sciences and Engineering, 2019, 41:61. 
15.Hussain, F.; Ellahi, R.; Zeeshan, Ah., Mathematical Models of ElectroMagnetohydrodynamic Multiphase Flows Synthesis with Nano-Sized Hafnium Particles, Appl. Sci. 2018,8, 275.

16. Sinha, A.; Shit, G.C., Electromagnet hydrodynamic flow of blood and heat transfer in a capillary with thermal radiation, Journal of Magnetism and magnetic materials, 2015,378,143-151.

17.Bhatti, M. M.; Sheikholeslami, M.; Zeeshan, Ah., Entropy Analysis on Electro-Kinetically Modulated Peristaltic Propulsion of Magnetized Nanofluid Flow through a Microchannel, Entropy. 2017, 19, 481.

18.Ranjit, N.K.; Shit, G.C., Entropy generation on electro-osmotic flow pumping by a uniform peristaltic wave under magnetic environment, Energy, 2017,128, 649-660

19.Hahm, J.; Balasubramanian, A.; Beskok, A., Flow and species transport control in grooved microchannels using local electrokinetic forces, AIP Publishing, Physic of Fluids , 2007,19, 013601.

20.Jhorar, R.; Tripathi, Dh.; Bhatti, M. M.; Ellahi, R., Electroosmosis modulated biomechanical transport through asymmetric microfluidic channel, Indian J Phys, published online ,2018.

21.Santiago, J. G. Electroosmotic Flows in Microchannels with Finite Inertial and Pressure Forces. Analytical Chemistry, 2001,73, 10.

22.Brown, A. B. D.; Smith, C. G.; Rennie, A. R. Pumping of water with an electric fields applied to asymmetric pairs of microelectrodes. Physical Review E, 2000,63, 016305.

23. Hadigol, M.; Nosrati, R.; Nourbakhsh, Ah., Raisee. Mehrd. Numerical study of electroosmotic micromixing of non-Newtonian fluids. Journal of Non-Newtonian Fluid Mechanics ,2011,166, 965-971.

24.Tripathi, Dh.; Yadav, A.u; Bég, O. A. Electro-osmotic flow of couple stress fluids in a micro-channel propagated by peristalsis. Eur. Phys. J. Plus ,2017, 132: 173.

25.Tripathi, Dh.; Bhushan, Sh.; Bég, O. A. Analytical Study of Electro-Osmosis Modulated Capillary Peristaltic Hemodynamics. Journal of Mechanics in Medicine and Biology ,2017,17, 5, 22.

26.Noreen, S.; Waheed, S.; Hussanan, Ab., Lu. D. Analytical Solution for Heat Transfer in Electroosmotic Flow of a Carreau Fluid in a Wavy Microchannel. Appl. Sci. 2019, 9, 4359.

27.Noreen, S.; Waheed, S.; Hussanan, A.; Lu D. Entropy Analysis in Double-Diusive Convection in Nanofluids through Electro-Osmotically Induced Peristaltic Microchannel. Entropy ,2019, 21, 986.

28.Ramesh, K.; Prakash, J. Thermal Analysis for Heat Transfer enhancement in Electroosmosis modulated Peristaltic transport of Sutterby Nanofluids in a Microfluidic Vessel. Journal of Thermal Analysis and Calorimetry, 2018.

29.Yavari, H.; Sadeghi, A.; Saidi, M. H.; Chakraborty, S. Temperature rise in electro-osmotic flow of typical non-Newtonian biofluids through rectangular microchannels. Journal of Heat Transfer, 2013.

30.Noreen, S.; Riaz, A.; Lu, D. Soret-Dufour effects in electroosmotic biorheological flow of Jeffrey fluid. Heat Transfer, Wiley Periodicals, Inc, 2020. 
31.Asghar, Z., ; Ali, N. Analysis of Mixed Convective Heat and Mass Transfer on Peristaltic Flow of Fene-P Fluid with Chemical Reaction. Journal of Mechanics, 2016,32, Issue 01, 83 92.

32.Waheed, S.; Noreen, S. Hussanan, A., Study of Heat and Mass Transfer in Electroosmotic Flow of Third Order Fluid through Peristaltic Microchannels. Appl. Sci. 2019, 9, 2164.

33.Prakash, J.; Sharma, A.; Tripathi, Dh. Thermal radiation effects on electro-osmosis modulated peristaltic transport of ionic nanoliquids in biomicrofluidics channel. Journal of Molecular Liquids, 2018,249, 843-855.

34. Vaidya, H., Rajashekhar, C.; Manjunatha, G.; Prasad, K. V.; Makinde, O. D.; Vajravelu, $\mathrm{K}$. Heat and mass transfer analysis of MHD peristaltic flow through a complaint porous channel with variable thermal conductivity. Phys. Scr. ,2020,95, $045219,11$.

35. Bandopadhyay, A.; Tripathi, Dh.; Chakraborty, S. Electro-osmosis-modulated peristaltic transport in microflu 